\title{
Kiefer- und Gesichtsschmerz - ein Fallbeispiel
}

Die funktionellen Zusammenhänge bei Patienten mit kraniomandibulären Beschwerden sind sehr komplex. Bei Frau G. löst eine vorbereitende Behandlung für eine Zahnkrone diffuse Schmerzen und Störungen aus. Ihre Manualtherapeutin fühlt ihr nicht nur auf den Zahn, sondern auch auf den N. maxillaris - und wandelt Dysfunktion in Funktion.

\section{Subjektiver Befund}

Frau G. ist 61 Jahre alt. Sie klagt über Schmerzen im rechten Kieferbereich und in der rechten Gesichtshälfte sowie über Ohrenund Zahnschmerzen. Insbesondere ihr oberer hinterer Molar und das Gebiet über dem rechten Arcus zygomaticus schmerzen ( Abb. 1). Außerdem flimmert ihr rechtes Auge, zeitweise tränt es auch. Die Beschwerden bestehen schon seit längerem. Ihre Schmerzmedikamente wirken inzwischen nicht mehr.

Die Beschwerden sind der Patientin bereits aus der Vergangenheit bekannt. Nach einer Zahnarztbehandlung vor vier Monaten traten sie wieder auf. Dort wurde sie auf das Einsetzen einer neuen Krone, 7. Molar oben rechts, vorbereitet.

Die Schmerzqualität beschreibt sie als scharf, brennend, ziehend und manchmal dumpf. Beginnen die Schmerzen, ist ihr Gesichtsbereich mitunter auch heiß. Auf der visuellen Analogskala stellt sie eine Schmerzintensität von 8,5 ein. Ihre Hobbys sind Wassergymnastik, Tennis und Walken. Die Patientin ist nicht berufstätig.

\section{Schmerzverhalten}

Bei äußeren Reizen wie Kälte und Wind nehmen die Schmerzen zu und entsprechen dann einer Neun auf der visuellen Analogskala. Das Gleiche gilt für Stress. Vor allem in stressigen Zeiten neigt die Patientin dazu, tagsüber die Zähne aufeinanderzupressen (Bracing) und nachts zu knirschen (Bruxismus). Deshalb trägt sie nachts eine orale Schiene.

Auf die Frage, ob es irgendetwas gibt, was den Schmerz reduzieren kann, gibt die Patientin an, dass Druck auf den Arcus zygomaticus die Schmerzen kurz lindert, insbesondere im Ohr. Gängige Schmerzmittel lindern die Beschwerden nicht. Bis vor kurzem war jedoch ein US-amerikanisches Rheumamittel hilfreich. Zum 24-Stunden-Verhalten: Der Schmerz tritt zwar nicht täglich auf, kann aber bis zu vier Tagen anhalten. Tageszeitliche Abhängigkeiten gibt es nicht.

\section{Vorgeschichte}

Die Patientin erinnert sich, dass sie sich als Kind den Kiefer „ausrenkte“ und danach den Mund nicht mehr so weit öffnen sollte. Mit etwa 25 Jahren begann eine massive Migräne, die sich vor allem auf die rechte Kopfseite konzentrierte. Hinzu kamen rechts Kiefer-, Ohr- und Zahnschmerzen, die den jetzigen Beschwerden entsprechen. Aufgrund der Beschwerden wurden die Weisheits- zähne gezogen, was aber die Symptome nicht beeinflusste. Einige Jahre später zu Beginn der Wechseljahre nahmen die Beschwerden ab, und die Migräne trat nicht mehr auf.

\section{Physikalische Untersuchung: Inspektion}

Bei erster Betrachtung der Patientin vermutet die Therapeutin eine Gesichtsasymmetrie, im Sinne einer Konvergenz auf der rechten Seite. Bei näherer Betrachtung und Messung bestätigt sich dies jedoch nicht ( Abb. 2). Die Patientin weist keine relevanten Gesichtsasymmetrien auf. Den ersten Eindruck einer anscheinend bestehenden Auffälligkeit löste eine HWS-Seitneigung nach rechts aus, ca. fünf Grad ( $\mathbf{A b b}$. 3). Außerdem hat die Patientin einen Schultertiefstand rechts. Der kraniozervikale Winkel beträgt 49 Grad ( $\bullet$ Abb. 4, $\triangleright$ Abb. 5).

\section{Neuromuskuläre Untersuchung: Kraniomandibuläre Region}

In der Untersuchung der kraniomandibulären Region fällt auf, dass die endgradige Depression mit 35 Millimetern und die Laterotrusion nach links mit 6 Millimetern eingeschränkt und schmerzhaft sind. Dagegen ist die Laterotrusion nach rechts mit 12 Millimetern unauffällig. Beim Blick in den Mund zeigen sich an den Zähnen Abrasionen, besonders rechts. Während der Palpation spürt die Therapeutin rechts lokale schmerzhafte Verhärtungen im M. masseter

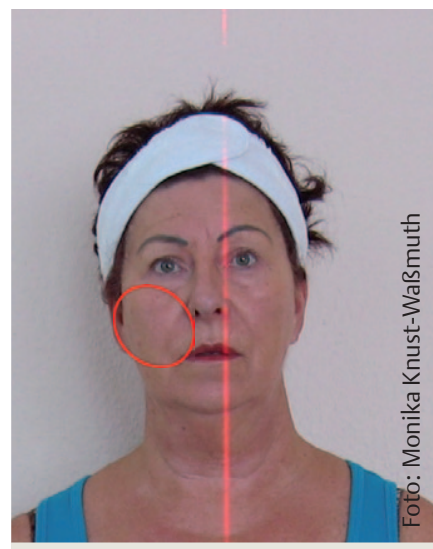

Abb. 1 Körpertabelle.

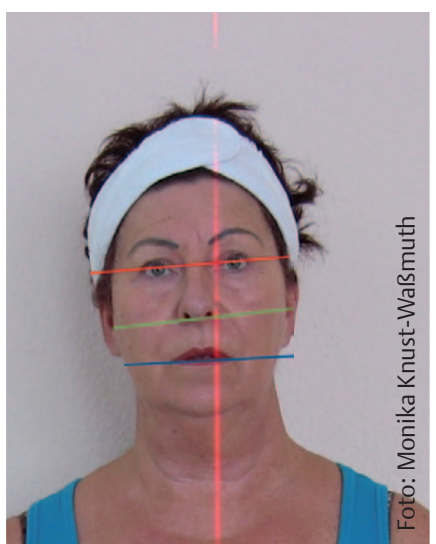

Abb. 2 Gesichtsmessung. 


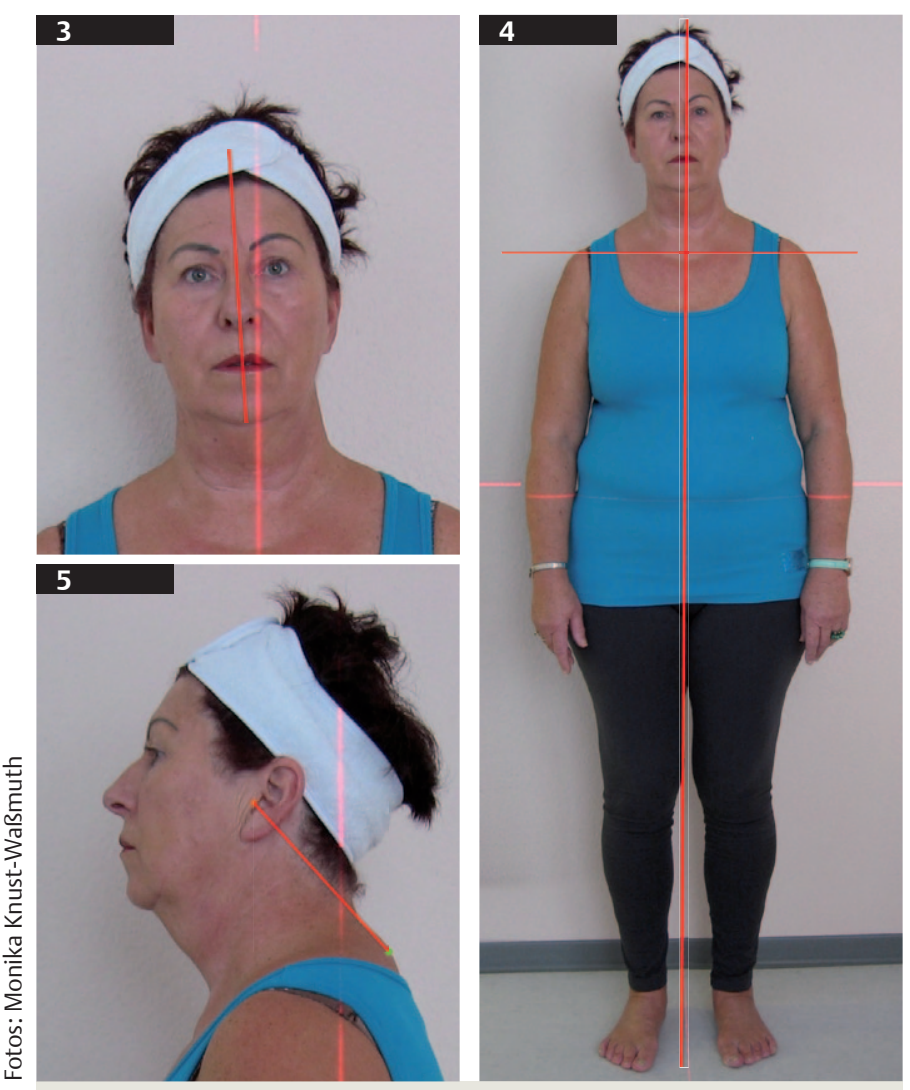

Abb. 3 HWS Seitneigung nach rechts.

Abb. 4 Leichter Schultertiefstand rechts.

Abb. 5 Kraniozervikaler Winkel. (Alle Messungen per CRAFTA-Klinimetrieprogramm.)

(Pars superficialis und profundus), M. temporalis anterior und M. pterygoideus medialis.

Clinical Reasoning: Vor allem Pressaktivitäten erhöhen die Aktivität der Kaumuskeln, des M. temporalis, M. masseter und des M. pterygoideus medialis [12]. Die abnormale Belastung kann auf Dauer lokale Entzündungsreaktionen in den Muskelfasern auslösen, die Travell und Simon als Triggerpunkte definieren [18]. Schmerztriggerpunkte enthalten eine erhöhte Konzentration verschiedener Neuropeptide wie die Substanz P (SP), den Tumor-Nekrose-Faktor $\alpha$ (TNF- $\alpha$ ), Interleukin-1 $\beta$ (IL-1 $\beta$ ) und viele weitere. Der phWert ihres Gewebes ist deutlich erniedrigt [16] und sie verursachen ausstrahlende Schmerzen. In diesem Fall strahlen die Triggerpunkte des M. masseter (Pars superficialis) in die Prämolaren und die Molaren des Oberkiefers aus sowie in den Sinus maxillaris. Die Triggerpunkte des M. pterygoideus medialis strahlen unter anderem zum Ohr und zum Kiefergelenk aus [18]. Es könnte ein Zusammenhang bestehen zwischen den Triggerpunkten der Patientin und ihren Schmerzen (Kiefer, Ohr, Jochbogen, Zähne).

Beim Blick in den Mund fallen rechts fehlende Zähne auf. Oben und unten sind jeweils der 7. und 8. Molar nicht (vollständig) vorhanden. Dies reduziert die Stützzone auf der rechten Seite. Die Patientin kaut feste Nahrung vorwiegend links.
Clinical Reasoning: Die Ausrichtung der maxillaren Okklusionsebene ist wesentlich für das kraniomandibuläre, -faziale und -zervikale Gleichgewicht. Erklärbar wird dies an den einwirkenden Kräften der Kaumuskulatur auf das Kranium. Der Unterkiefer ist kranial beidseits mit dem Os temporale verknüpft und ebenso mit dem Os parietale, Os zygomaticus, Os sphenoidale und der Maxilla. Über das Hyoid ist er kaudal mit Brustkorb und Schultergürtel verbunden. Man nimmt an, dass eine unsymmetrische Kraftübertragung der Kaumuskulatur aufgrund einer schiefen Okklusionsebene die Funktion neuraler Strukturen stört. Eine unphysiologische Belastung der Schädelbasis und die damit verbundene Beweglichkeitseinschränkung der Suturen beeinträchtigen die Liquorzirkulation im Gehirnund Rückenmark [20]. Nach dem kranioosteopathischen Modell unterstützen die Kräfte der Kaumuskulatur die Flexions- und Extensionsbewegung der sphenobasillären Synchondrose. Sie tragen so zu einer Stabilisierung des harmonischen kranialen Bewegungsrhythmus bei $[3,17]$. Die fehlende Stützzone könnte somit das neurale, kraniale und mandibuläre System der Patientin beeinflussen und ihre Dysfunktionen auslösen oder unterhalten.

\section{Neuromuskuläre Untersuchung: Kraniofaziale Region}

In der allgemeinen Untersuchung des Viszerokraniums ist das Os zygomaticum auffällig. Die spezifische Untersuchung des Os zygomaticum mit dem Os temporale reproduziert die Beschwerden der Patientin ( Abb. 6). Dabei kann der Schmerz durch einen Bewegungsimpuls des Os zygomaticum nach kranial bei fixiertem Os temporale wiederholt werden - und umgekehrt mit einem Bewegungsimpuls des Os temporale nach kaudal bei fixiertem Arcus zygomaticus.

\section{Neurodynamische Untersuchung: Kraniales Nervensystem}

Während der passiven hochzervikalen Nackenflexion (erste Kategorie; Tab. 1, \ Abb. 7) beziffert die Patientin ihren Schmerz mit 8,5 auf der visuellen Analogskala. Beim Test der zweiten Kategorie ist der N. maxillaris auffällig: Die Fingerrollbewegung kaudal des

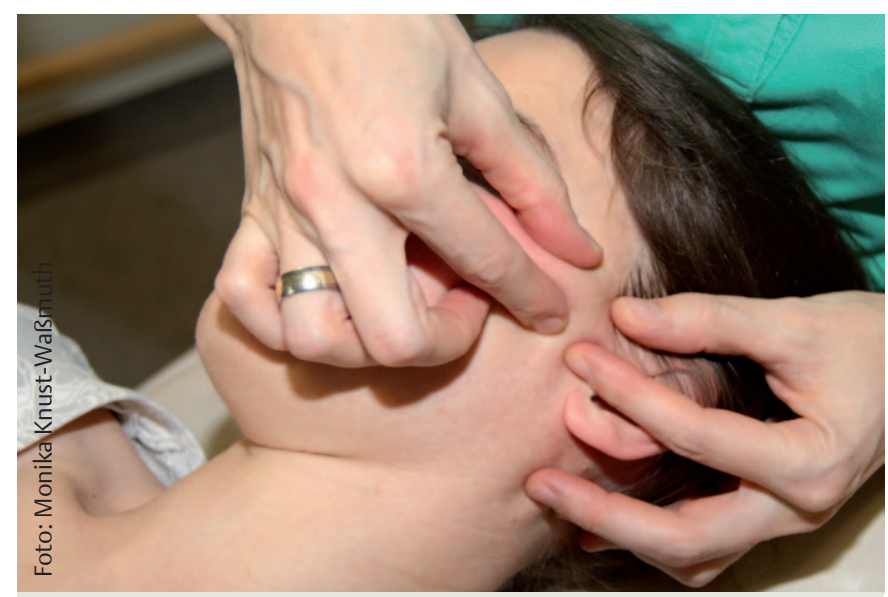

Abb. 6 Spezifische Untersuchung des Os zygomaticum mit dem Os temporale. 


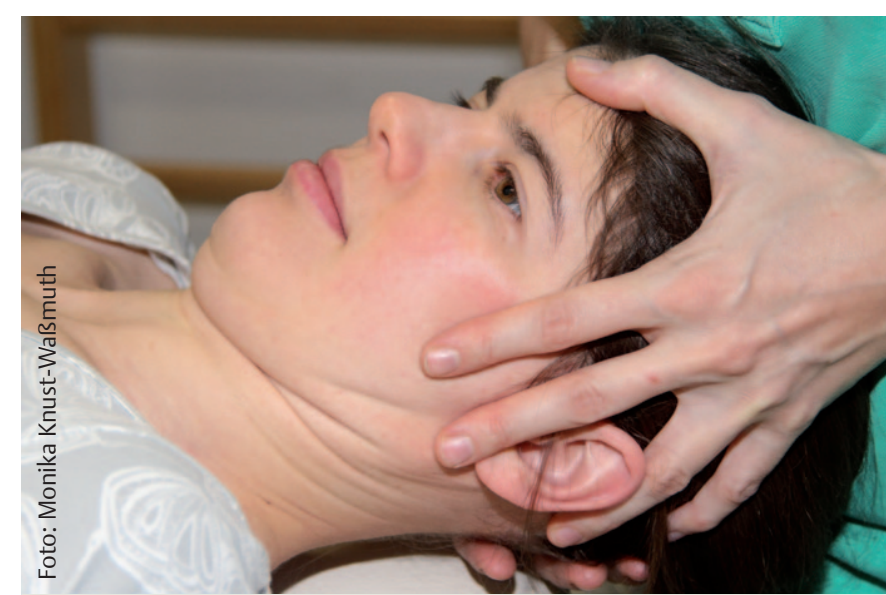

Abb. 7 Hochzervikale Nackenflexion.

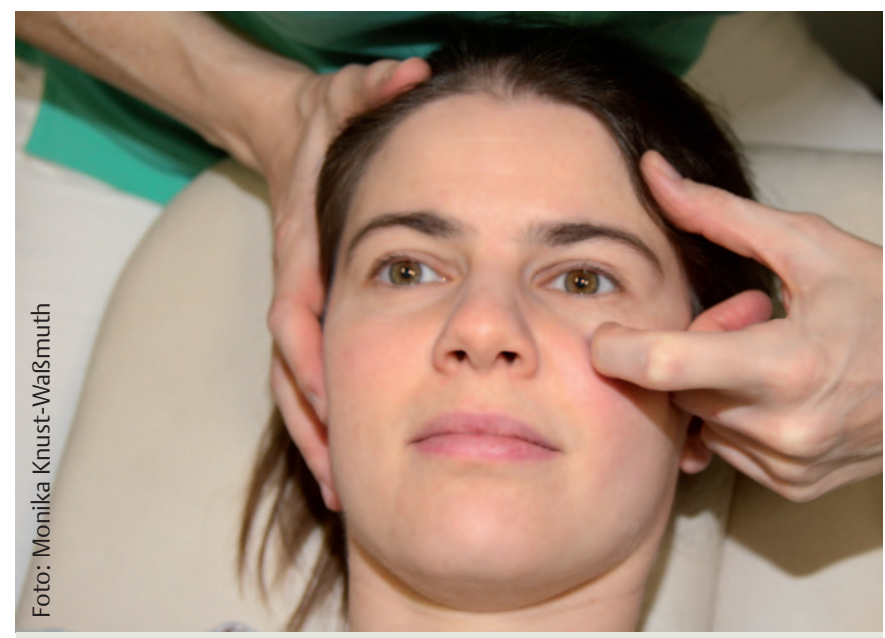

Abb. 8 Palpation N. infraorbitalis am Foramen infraorbitalis.

rechten Auges nach kranial reproduziert den Schmerz der Patientin und ebenso die Palpation des N. infraorbitalis am Foramen infraorbitale $(\triangleright$ Abb. 8).

Clinical Reasoning: Für die Untersuchung und Behandlung des kranialen Nervensystems ist eine spezielle Ausbildung erforderlich. Die dort vermittelten manualtherapeutischen Verfahren eignen sich insbesondere für die Therapie von Patienten mit Kiefer-, Kopf- und Gesichtsschmerzen. Gesichtsschmerzen verursacht meistens der N. trigeminus (V. Hirnnerv), der gegebenenfalls untersucht und behandelt wird. In diesem Fall sind die Beschwerden vor allem im Versorgungsgebiet des N. maxillaris lokalisiert ( Tab. 2). Deshalb beschließt die Therapeutin ihn in Anlehnung an den standardisierten neurodynamischen Test des N. mandibularis [19] zu untersuchen.

Untersuchung N. maxillaris: Die Therapeutin kombiniert eine hochzervikale Flexion der HWS mit einer Lateroflexion, kontralateral zu der zu untersuchenden Seite, und erhöht so die Spannung des Nervenursprungs in Hirnstammnähe. Augenbewegungen nach kranial und me-
Tab. 1 Kraniale Nerven: Untersuchung der Neurodynamik nach drei Hauptkategorien [20].

\begin{tabular}{|c|c|}
\hline Kategorie & Test \\
\hline 1 & - Nackenflexion und -extension \\
\hline 2 & $\begin{array}{l}\text { - N. trigeminus (V2 N. maxillaris) } \\
\text { - N. facialis } \\
\text { - } \mathrm{N} \text {. accessorius } \\
\text { - } \mathrm{N} \text {. hypoglossus }\end{array}$ \\
\hline 3 & $\begin{array}{l}\text { - N. olfactorius } \\
\text { - N. opticus } \\
\text { - N. oculomotorius } \\
\text { - N. trochlearis } \\
\text { - N. abducens } \\
\text { - N. vestibulocochlearis } \\
\text { - N. glossopharyngeus } \\
\text { - N. vagus }\end{array}$ \\
\hline
\end{tabular}

Tab. 2 N. maxillaris.

\begin{tabular}{|l|l|}
\hline Äste & Versorgungsgebiet \\
\hline $\begin{array}{l}\text { Rr. ganglionares } \\
\text { ad ganglion } \\
\text { pterygopalatinum }\end{array}$ & $\begin{array}{l}\text { - Schleimhaut der Nasenmuscheln, der } \\
\text { Siebbeinzellen, des harten und weichen } \\
\text { Gaumens }\end{array}$ \\
\hline N. zygomaticus & $\begin{array}{l}\text { - Haut über dem Jochbein und im vorderen } \\
\text { Schläfenbein } \\
- \text { viszeromotorische Tränendrüse über pa- } \\
\text { rasympathische Fasern aus dem N. facia- } \\
\text { lis, die er zum N. lacrimalis abgibt }\end{array}$ \\
\hline N. infraorbitalis & $\begin{array}{l}\text { Wange zwischen Unterlid und Oberlippe } \\
\text { - Schleimhaut des Sinus maxillaris } \\
\text { - Oberkieferzähne }\end{array}$ \\
\hline
\end{tabular}

dial erhöhen die Belastung des zygomatikotemporalen Astes. Kraniale Bewegungen am Os zygomaticum bringen die zygomatikofazialen Äste unter Spannung. Bewegungen der Maxilla belasten die infraorbitalen Äste und Bewegungen am Palatinum die palatinen Äste [20].

\section{Behandlung}

Zunächst behandelt die Therapeutin die kraniofaziale Region und das kraniale Nervensystem, da die neurodynamische Untersuchung hier die Schmerzen der Patientin reproduzierte.

Kraniofaziale Region: Die Therapie beinhaltet kraniofaziale Techniken der mechanischen Berührungsflächen des N. maxillaris. Durchgeführt werden schwerpunktmäßig Techniken des Os zygomaticum kombiniert mit dem Os temporale. Zusätzlich führt die Therapeutin faziale Techniken der Maxilla mit dem Os sphenoidale und des Os zygomaticum aus, einseitige Maxillatechniken, Behandlungen des Os zygomaticum mit dem Os frontale sowie des Os sphenoidale mit dem Os palatinatum und Os sphenoidale mit dem Os occipitale.

Kraniales Nervensystem: hochzervikale Nackenflexion zur neuralen Mobilisation des N. maxillaris und Palpationstechniken für den $\mathrm{N}$. infraorbitale [5]. 
Kraniomandibuläre Region: Die Behandlung umfasst Techniken zur Wiederherstellung der gestörten Kieferfunktion sowie funktionelle Weichteiltechniken für die hypertone Kaumuskulatur rechts $[7,11]$. Die verminderte Kiefergelenkbeweglichkeit rechts in Richtung Depression und Laterotrusion wird mit Traktion und Translation behandelt $(\checkmark$ Abb. 9$)$.

Clinical Reasoning: Die Traktion des Kiefergelenks ist eine bewährte Technik in der CMD-Therapie $[6,9,13]$. Sie separiert die Gelenkpartner $[14,15]$ und zielt auf den Erhalt oder die Verbesserung der Gelenkbeweglichkeit ab sowie auf die Schmerzlinderung im Gelenk [4]. Die Wirkung der Traktion und Translation besteht aus einer Dehnung bei Schrumpfungen des Kapsel-Band-Apparates und Muskelverkürzungen und entlastet die Gelenkflächen [8].

Kaumuskeln: Auf den Triggerpunkten werden transversale Bewegungen durchgeführt - mit und ohne Bewegung des jeweiligen Muskels ( Abb. $10-$ Abb. 12). Ziel dieser Weichteiltechniken ist, die afferenten Inputs und Verarbeitungsprozesse zu verbessern [2].

\section{Information und Eigentraining}

Die Patientin erhält eine Einweisung in das Habit Reversal Training, ein verhaltenstherapeutisches Verfahren gegen unphysiologische, nervöse Gewohnheiten [1]. Sie verringert so die Bruxismusaktivität. Außerdem lernt sie, wie sie die hypertone Kaumuskulatur selbstständig behandelt und erhält koordinative Übungen, um die Kiefermotorik zu verbessern [7, 11].

\section{Verlauf}

Es folgen acht Behandlungseinheiten im wöchentlichen Abstand. Im Laufe der Behandlungszeit sinkt der anfängliche Schmerz auf der visuellen Analogskala von 8,5 auf null. Um den langfristigen Behandlungserfolg zu prüfen, wird die Patientin zwei Monate nach der letzten Behandlungseinheit angerufen. Ihre Beschwerden sind immer noch deutlich reduziert. Der Schmerz trat nur zweimal unterschwellig auf.

\section{Reflexion}

Für ein positives Testergebnis einer neurogenen Quelle sprechen: Symptomreproduzierbarkeit, ein Rechts-links-Unterschied in der strukturellen Differenzierung sowie übereinstimmende subjektive und physikalische Daten [2]. In diesem Fall reproduzierten kraniofaziale Techniken der zygomatischen Region sowie allgemeine neurale und spezifische Tests des N. maxillaris die Symptome. Ein Rechts-links-Unterschied war vorhanden, die subjektiven und physikalischen Daten wie Vorgeschichte, Schmerzverhalten und -qualität passten zu einer neurogenen Quelle, sodass die Therapeutin die Hypothese einer Funktionsstörung des N. maxillaris aufstellte. Auch das retrospektive Assessment untermauerte diese Hypothese. Möglicherweise bildeten sich durch die Zahnextraktion des 7. und 8. Molaren rechts AIGS (Abnormal Impulse Generating Sites) des N. maxillaris. Reize wie Kälte, Wind und Stress aktivierten sie und verursachten den Schmerz.
Die kraniomandibuläre Region ist eine weitere Quelle. Durch die Luxation des rechten Kiefergelenkes im Kindesalter und die anschließende Angst, den Kiefer maximal zu öffnen, könnte sich eine kraniomandibuläre Dysfunktion entwickelt haben. Die extrahierten Molaren beziehungsweise die fehlende Stützzone und die Bruxismusaktivität unterhielten sie. Aufgrund der funktionel-

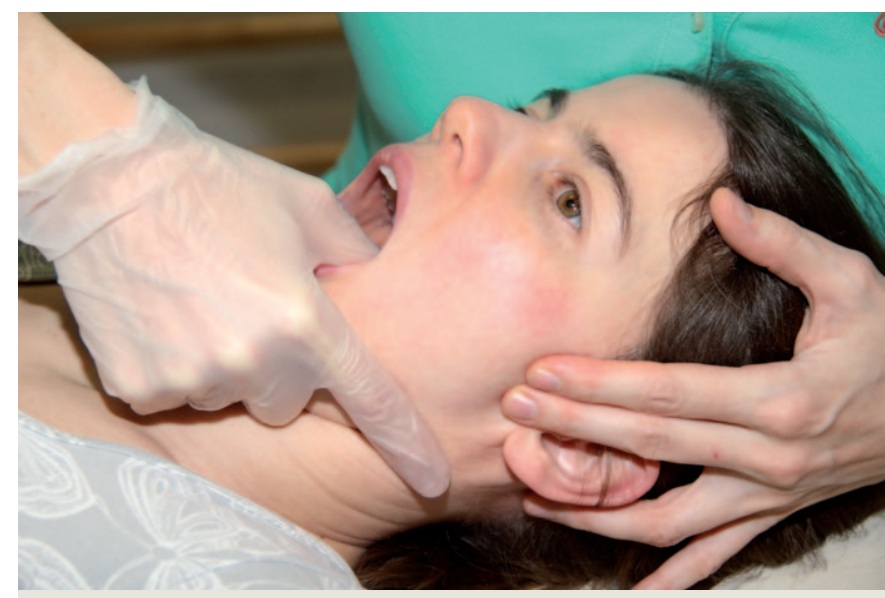

Abb. 9 Traktion des Kiefergelenks bei maximaler Mundöffnung.
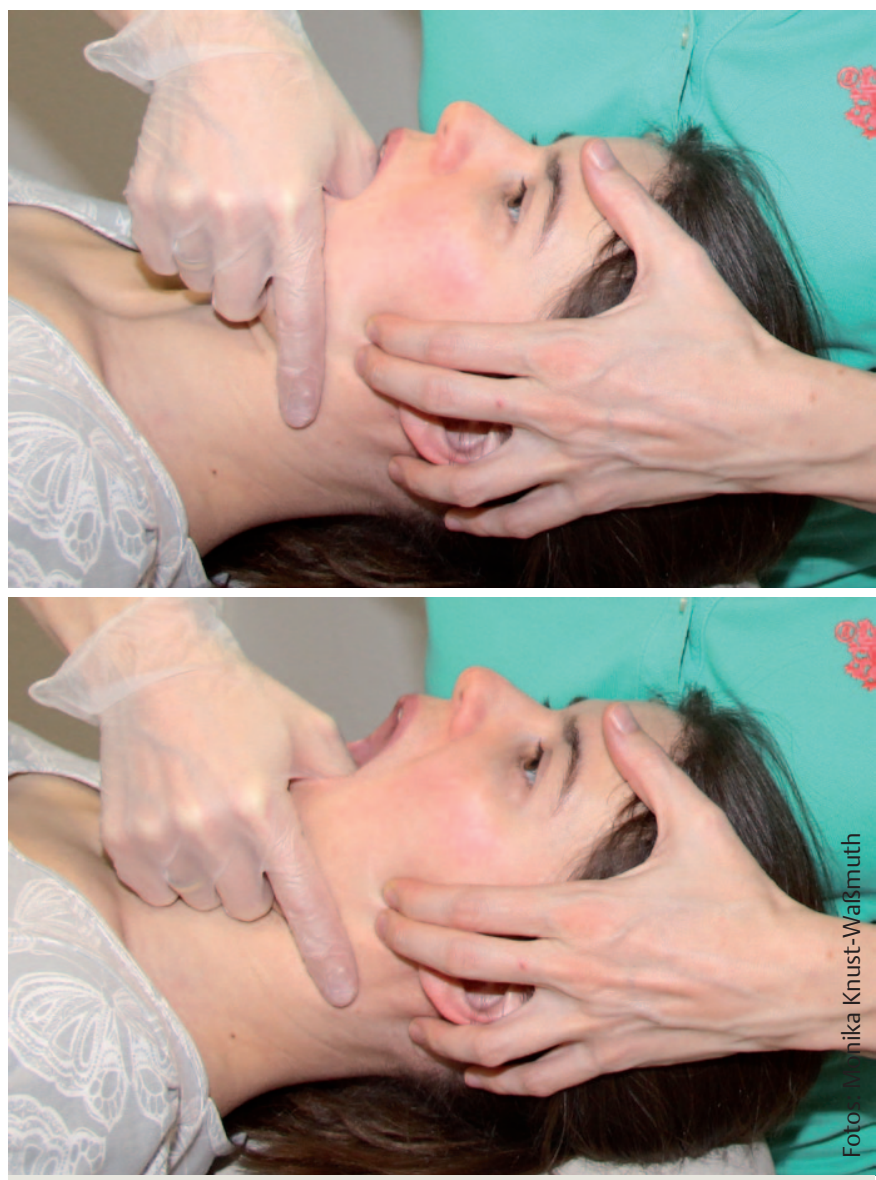

Abb. 10 Triggerpunktbehandlung der Pars superficialis des M. masseter mit Bewegung. 
len Verbindungen, unter anderem mit dem kraniofazialen System, könnte die kraniomandibuläre Dysfunktion ein beitragender Faktor zu den migräneartigen Beschwerden gewesen sein. Peripher neurogene Störungen verknüpfen sich oft mit früheren neuropathischen Störungen. Die Zahnarztbehandlung wirkte wahrscheinlich als wiederauslösender Faktor.
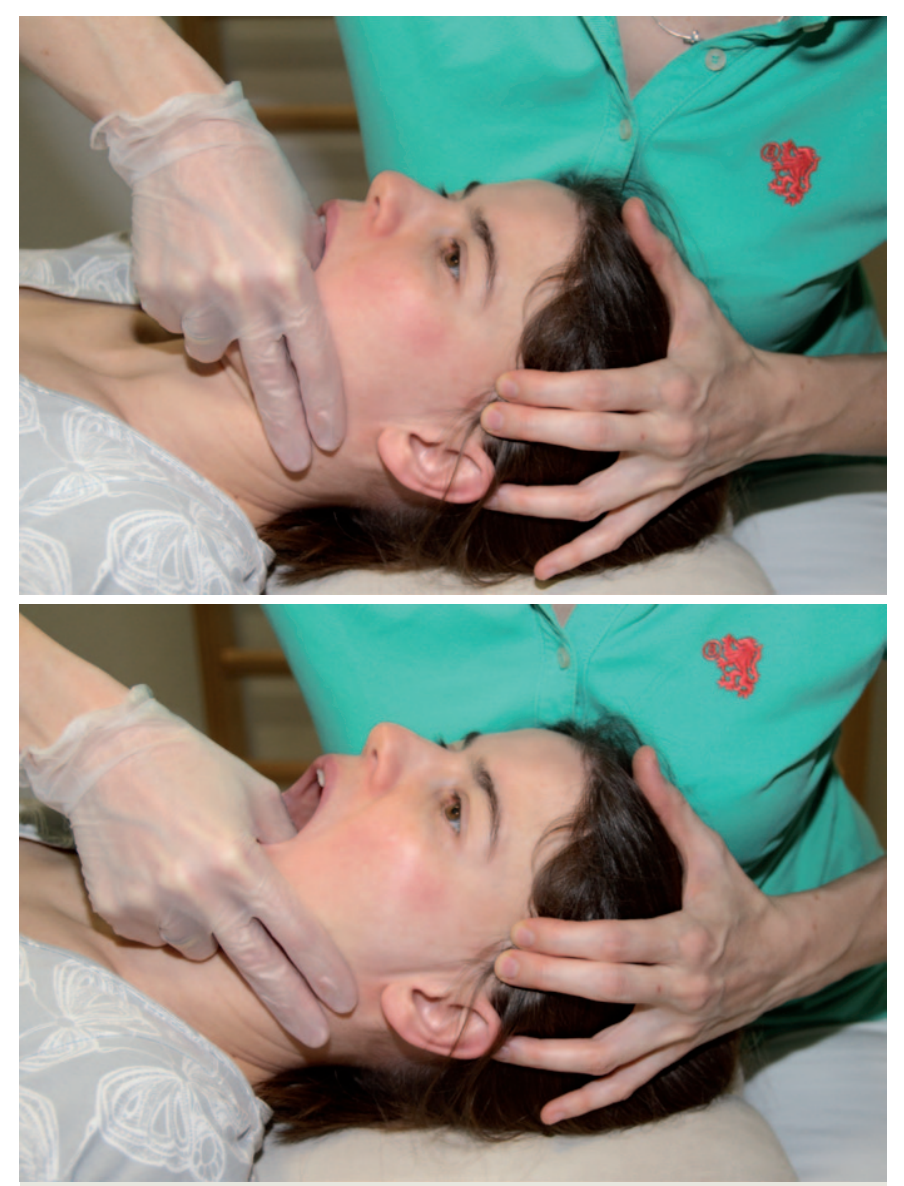

Abb. 11 Triggerpunktbehandlung der Pars anterior des M. temporalis mit Bewegung.

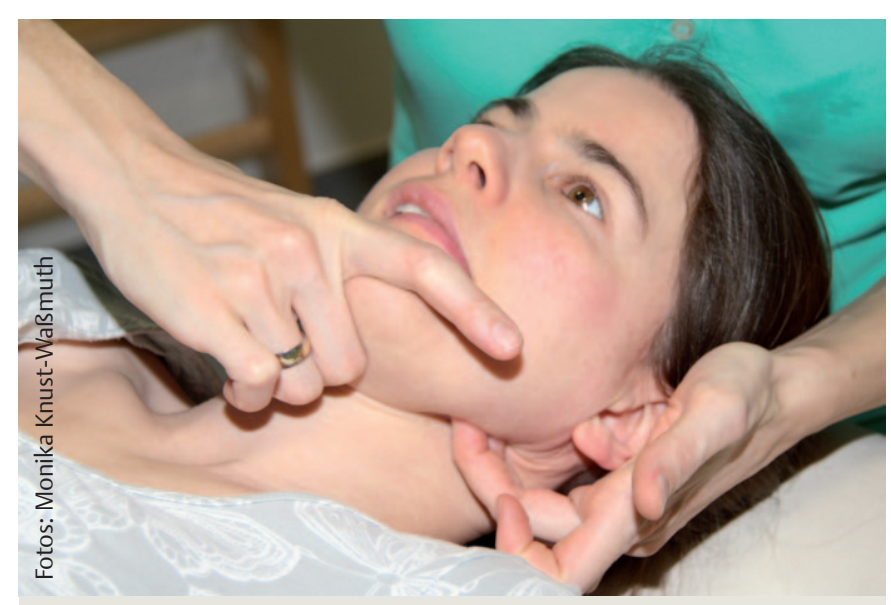

Abb. 12 Triggerpunktbehandlung M. pterygoideus medialis.

\section{HABIT REVERSAL TRAINING: BEISPIEL BRUXISMUS UND BRACING}

Diese verhaltenstherapeutische Methode nach Azrin und Nunn zielt darauf ab, gewohnheitsmäßige, unbewusste Verhaltensketten zu unterbrechen. Zunächst informiert der Therapeut die Patientin über die mechanischen Folgen des Bruxismus und Bracings wie Zahnschäden, Überlastungen des Halteapparates, der Kaumuskulatur, Schmerzen und so weiter. Ist der Patient zur Verhaltensänderung motiviert, wird seine Selbstbeobachtung gefördert. Er zeichnet das unerwünschte Verhalten in Tagebüchern oder Protokollen auf und notiert Situationen, in denen er knirscht oder presst. Als Nächstes übt der Patient eine alternative Handlung ein. Beispiel: Die Faust unter das Kinn halten und in die Mundöffnung, gegen die Faust, anspannen - so lange, bis das Gefühl, die Zähne zusammenbeißen zu müssen, verschwunden ist. Diese Handlungsalternative setzt der Patient so oft wie möglich in den von ihm beschriebenen Situationen ein. Nach und nach ersetzt das neue Verhalten das unphysiologische. Das Training ergänzt die orale Schiene, die meist nachts getragen wird.

\section{FAZIT} (

Aufgrund der langen Vorgeschichte ist neben der kraniofazialen Behandlung die Therapie auf der Verarbeitungsebene ein wesentlicher Baustein. Daher erlernte die Patientin das Habit Reversal Training. Auch der durch die muskulären Weichteiltechniken geänderte afferente Input und bewusste koordinative Kieferbewegungen sind wesentlich für die Reorganisation der kraniomandibulären Region im sensomotorischen Kortex. Auf die zervikale Region wurde bei dieser Patientin nicht näher eingegangen, da sie keine schmerzauslösenden oder -reproduzierenden Zeichen aufwies.

Literaturverzeichnis am Ende der HTML-Version unter www.thieme-connect.de/products/manuelletherapie

\section{AUTORIN}

Monika Knust-Waßmuth, BSc, Manualtherapeutin Die Absolventin der Hochschule Osnabrück gewann mit ihrer Bachelorarbeit zum Thema kraniomandibuläre Dysfunktion den IFK-Wissenschaftspreis und ist seit fast zehn Jahren zertifi-

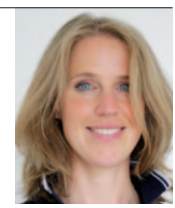
zierte CRAFTA-Therapeutin und -Assistentin. Neben der praktischen Arbeit hält sie Vorträge unter anderem zum Thema Kopf-, Kiefer- und Gesichtsschmerz.

Kalkofenweg 6

55130 Mainz

monika.knust@web.de

\section{BIBLIOGRAFIE}

DOI 10.1055/s-0035-1557155

manuelletherapie 2015; 19: 121-125

(c) Georg Thieme Verlag KG

Stuttgart · New York · ISSN 1433-2671 